\title{
Clinical and Immunohistochemical Features of Intracardiac Leiomyoma in a Dog
}

\section{Hyun-Woo Yoon ${ }^{\dagger}$ \\ Keon $\mathrm{Kim}^{\dagger}$ \\ Jun-Young Park ${ }^{\dagger}$ \\ Min-Soo Kim \\ Jae-Beom Ju \\ Chang-Min Lee* \\ Guk-Hyun Suh*}

Department of Veterinary Internal Medicine, College of Veterinary Medicine and BK21 FOUR Program, Chonnam National University, Gwangju 61186, Korea

${ }^{\dagger}$ Hyun-Woo Yoon, Keon Kim, and Jun-Young Park contributed equally to this work.

*Correspondence: cmlee1122@jnu.ac.kr (Chang-Min Lee), ghsuh@jnu.ac.kr (Guk-Hyun Suh)

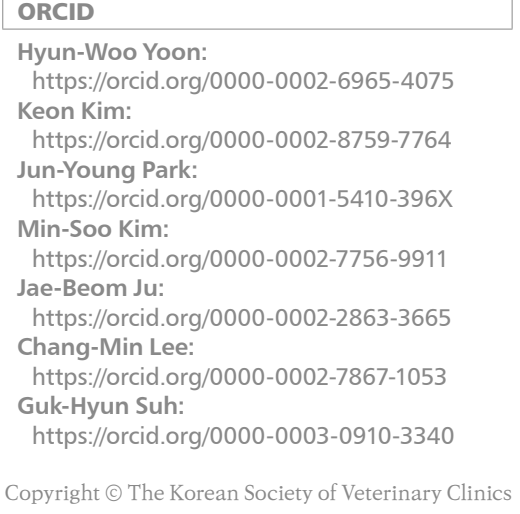

Abstract A 10-year-old spayed female Yorkshire terrier dog with a history of pleural effusion and dyspnea for a week was referred to the Veterinary Teaching Hospital. Radiography revealed pulmonary edema and pleural effusion. A mass infiltrating the right atrial lumen and especially the supra-tricuspid valve was identified with echocardiography. The mass was diagnosed as intracardiac neoplasia. Symptomatic treatment was prescribed to alleviate the symptoms of heart failure. Despite symptomatic treatment, the patient died a few days later. After obtaining consent from the owner, necropsy and histopathological evaluation were performed. The result was consistent with cardiac leiomyoma, and the diagnosis was confirmed by immunohistochemical staining. To the authors' knowledge, this case is the first report of intracardiac leiomyoma in a dog in Korea.

Key words cardiac tumor, dog, intracardiac leiomyoma, Korea. 


\section{Introduction}

Cardiac tumors in dogs are rare. In 1999, Ware and Hopper reported 1,383 dogs with cardiac tumors from a total of 729,265 dogs (19). Although the incidence rate of cardiac tumors is only $0.19 \%$, they can have critical effects on the cardiovascular system. Cardiac tumors in dogs can be categorized according to their histological features. Hemangiosarcoma is the most frequently observed cardiac tumor in dogs. Hemangiosarcoma can arise from any part of the body with a vascular structure, but the spleen is the most frequent site of primary lesion of hemangiosarcoma, followed by the right atrium and liver $(2,8)$. The aggressive and malignant features of hemangiosarcoma (12) may easily lead to metastasis to various organs of the body, among which the lungs are the most frequently affected (17). Aortic body tumors have also been reported in dogs; however, their incidence is 10-fold lower than that of hemangiosarcoma. Aortic body tumors are tumors of chemoreceptor tissue, located at the aortic root or heart base (19).

In humans, other cardiac tumors including leiomyoma have been reported. Leiomyoma is a slow-growing, benign, non-invasive neoplasm of smooth muscle. As smooth muscle is widely distributed throughout the body, leiomyoma can arise from any part of the body. Leiomyoma in humans can arise from the uterus and in rare cases, it might proliferate to the right heart (13). As in humans, the incidence of primary cardiac leiomyoma arising from the right heart in dogs is very low. The first case of primary cardiac leiomyoma in a 1-yearold intact female Labrador retriever was reported by Gallay et al. (5) in the United States, with the main symptom being sudden syncope caused by bradycardia. Here we present the first canine case of intracardiac leiomyoma in Korea.

\section{Case Report}

A 10 years old, spayed female Yorkshire terrier dog with a history of severe pleural effusion and dyspnea for a week was referred from the local animal hospital to the Veterinary Medical Teaching Hospital, Chonnam National University. The patient's body weight was $3 \mathrm{~kg}$. The dog showed anorexia, lethargy, dyspnea, and panting (60 breaths per min). Systolic blood pressure was $160 \mathrm{mmHg}$ and rectal temperature was $38.5^{\circ} \mathrm{C}$. Cardiac auscultation revealed a muffled heart sound.

Laboratory abnormalities included a moderate leukocytosis (leukocyte count $22.2 \times 10^{3} / \mathrm{L}$, reference range 4.0-15 $\times 10^{3}$ / L) and moderate granulocytosis (granulocyte count $18.1 \times$ $10^{9} / \mathrm{L}$, reference range $\left.7.3-12.6 \times 10^{9} / \mathrm{L}\right)$. Serum biochemistry results were unremarkable. Urinalysis revealed urine specific gravity of 1.030. In addition, the urine dipstick test was normal.

Radiographic examination revealed a pleural effusion, pulmonary edema, and an obscured cardiac silhouette (Fig. 1A). Echocardiographic examination revealed the presence of a mass in the heart and deformation of both atriums due to the mass. The mass appeared to be located on the valve side of the heart (Fig. 1B).

Based on the clinical findings, blood test results, and diagnostic imaging results, thoracocentesis was performed to remove the pleural fluid. Total protein and glucose levels in the pleural fluid were $3.5 \mathrm{~g} / \mathrm{dL}$ and $200 \mathrm{mg} / \mathrm{dL}$, respectively, and the pleural fluid was classified as a modified transudate. After determining that there was a problem with the blood circulation in the heart, medication was prescribed to relieve the symptoms of the patient: furosemide ( $1 \mathrm{mg} / \mathrm{kg} \mathrm{PO} \mathrm{q} 12 \mathrm{~h}$ ), enalapril $(0.5 \mathrm{mg} / \mathrm{kg}$ PO q $12 \mathrm{~h})$, and amlodipine $(0.2 \mathrm{mg} / \mathrm{kg}$ $\mathrm{PO}$ q $12 \mathrm{~h}$ ). The clinical signs were improved through medi-
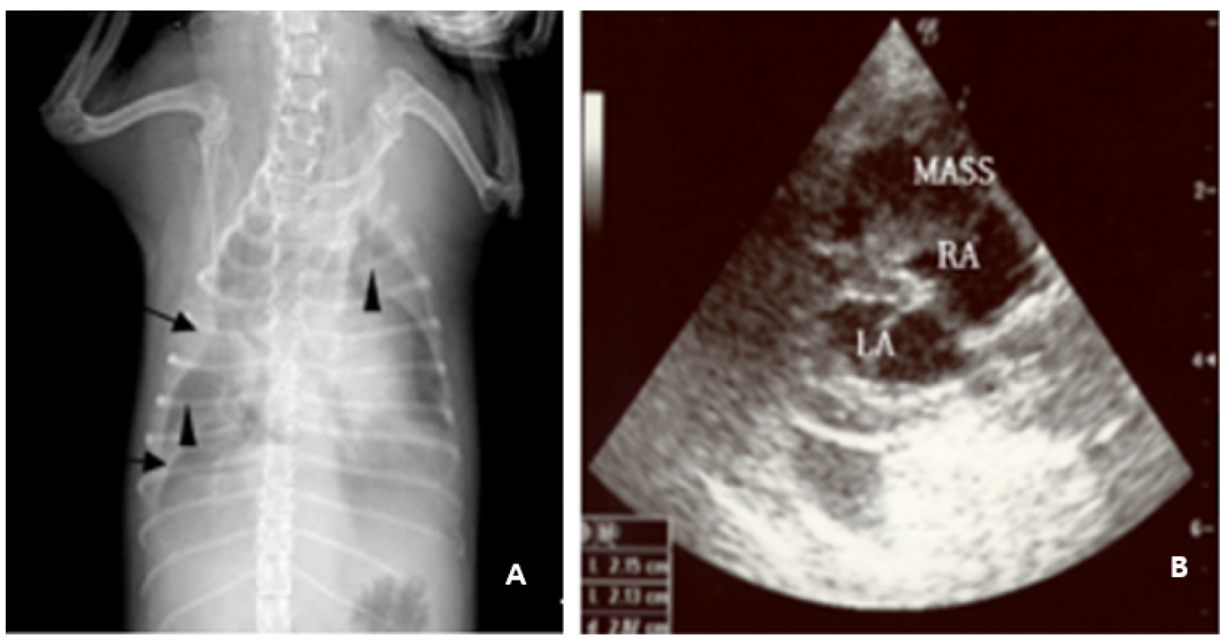

Fig. 1. Radiograph and echocardiographs of the patient. (A) Dorsoventral radiograph. The thickness of the pleural wall seems to be increased owing to severe pleural effusion (arrow). Evaluation of cardiac structure revealing a cardiac silhouette sign that is prominent on account of pulmonary edema and collapse (arrowhead). (B) Right long-axis four-chamber view. The size of the mass is $21 \mathrm{~mm} \times 21 \mathrm{~mm}$. A mass infiltrating the right atrial (RA) lumen is attached to the tricuspid valve. Approximately $60 \%$ of the right atrial lumen is occupied by the mass and structure of the RA, and the $L A$ is destructed. $R A$, right atrium; $R V$, right ventricle; LA, left atrium. 
cation and thoracocentesis, but the patient suddenly died 15 days after the first treatment.

A necropsy was conducted with the owner's approval. The necropsy revealed severe pleural fluid, severe diffuse pulmonary congestion, and edema. Gross examination of the heart revealed a firm, white, and ovoid-shaped mass in the right atrium (Fig. 2A). A cross-section of the mass showed a white structure and a few hemorrhages (Fig. 2B). When the abdominal organs were examined grossly, severe diffuse mottled lesions in the liver were found prominently.

The intracardiac mass of the right atrium was evaluated histopathologically. The tumor cells had a typical histoid pattern, and the cells were arranged toward various directions in interlacing patterns with infiltration of neutrophils and macrophages. The mass seemed to be a stromal tumor accompanied by pyogranulomatous inflammation with necrosis in the epicardial area (Fig. 3).

Histopathologic evaluation of other organs was performed. Evidence of metastasis was not detected in other organs. The lung tissue showed severe multiple alveolar emphysema and mild-to-moderate multiple congestions, whereas mild-to-moderate multiple congestions and swelling were observed in the liver.

Based on necropsy and histopathological findings, heman- giosarcoma, leiomyoma, desmoid tumor, rhabdomyosarcoma, and malignant fibrous histiocytoma were found to constitute the mass. Immunohistochemistry is indispensable to clarify the components of the mass.

Immunohistochemical slides were evaluated microscopically to determine the extent of positively stained tumor tissue, which was scored as 0 (negative), 1 ( $<25 \%$ positive), 2 (26$50 \%$ positive), or 3 ( $>50 \%$ positive). In the immunohistochemical identification, brown staining indicated a positive result, and no staining indicated a negative result. Immunohistochemical identification of the white mass in the right atrium was negative for cytokeratin (score: 0 ) but positive for vimentin (score: 3), desmin (score: 2), and smooth muscle actin (score: 2) (Fig. 4). Based on the immunohistochemical staining and observation of the spindle-shaped cells of the intracardiac mass of the right atrium, a diagnosis of intracardiac leiomyoma was achieved.

\section{Discussion}

According to a study conducted in 1999, the incidence of cardiac tumors in dogs is $0.19 \%$, accounting for a low percentage of all canine tumors in the United States (19). Hemangiosarcoma is the most frequently observed among
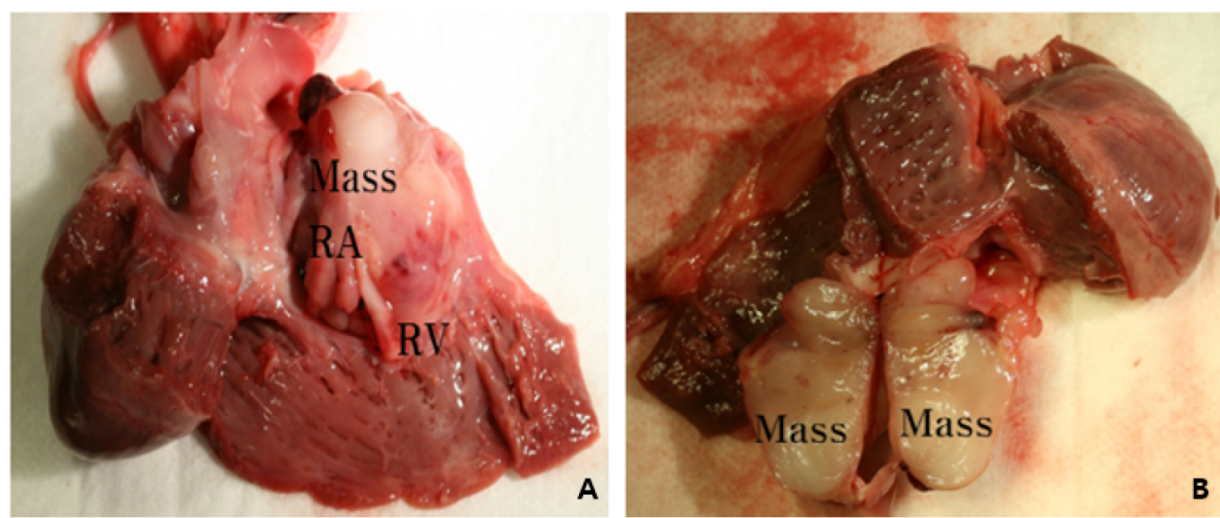

B

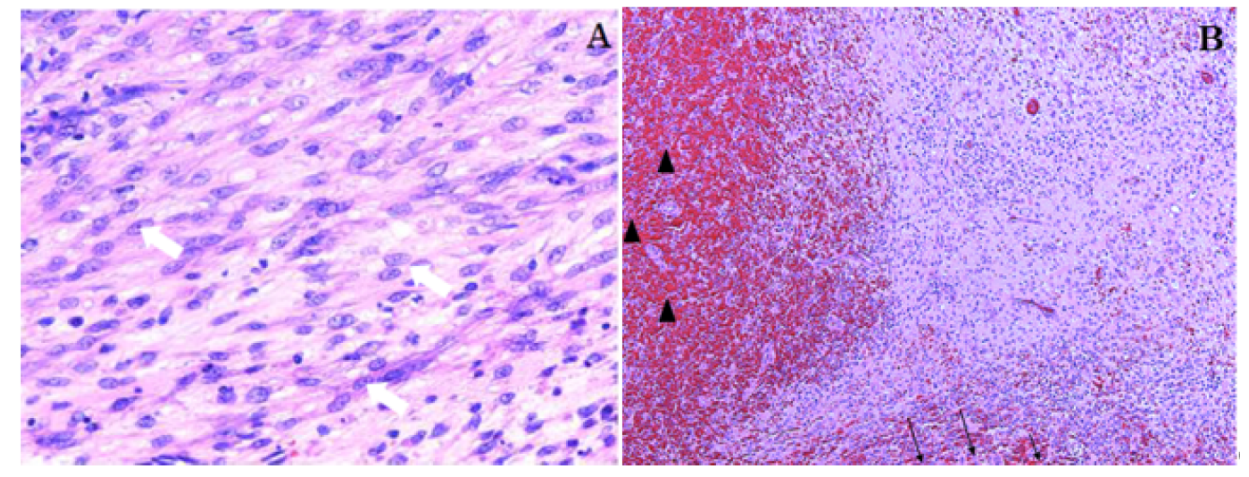

Fig. 3. Histopathological findings of the mass and surrounding tissue. Hematoxylin and eosin staining. Longitudinal section of the mass (white arrow, A. 200x) showing spindle cells with blunt-ended nuclei with the characteristics of smooth muscle cells. Pyogranulomatous inflammation (black arrow, B, 50×), congestion, and necrosis (arrowheads, B, 50×) in the epicardial area invaded by the tumor are shown above. 


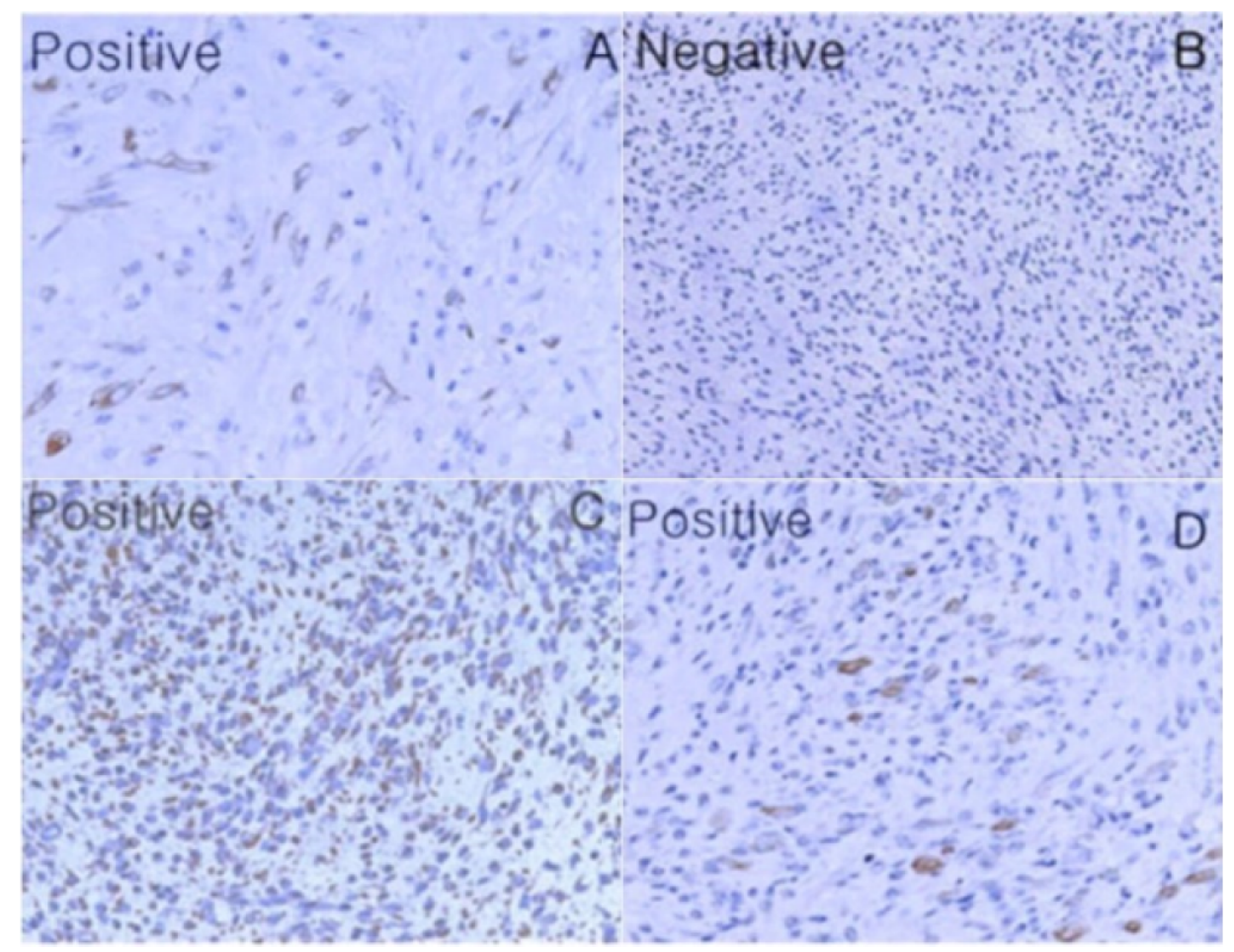

Fig. 4. Immunohistochemical staining of the mass. Positive results are indicated by brown-stained tissue. Smooth muscle actin (A), cytokeratin (B), vimentin (C), and de$\operatorname{smin}(D)$. all cardiac tumors, followed by Aortic body tumors $(2,4,7)$. Other tumors that can affect the canine heart include mesothelioma, myxoma, myxosarcoma, fibroma, fibrosarcoma, rhabdomyoma, and rhabdomyosarcoma $(10,11,14)$. Meanwhile, cardiac leiomyoma in dogs has rarely been reported. Similar to this case, primary cardiac leiomyoma with an advanced atrioventricular block was reported in a 1-year-old intact female Labrador retriever dog (5). In general, leiomyoma in dogs is known to occur mainly in the genital tract, especially the lower genital tract, including the vagina and vulva (1). In addition, it is known that leiomyoma can be detected in the gastrointestinal tract, urethra, and third eyelids (9). Further, it is known that infiltration to the heart can occur secondary to intracardiac leiomyomatosis and that smooth muscle cells proliferate in blood vessels when leiomyomatosis occurs. Importantly, if the cells reach the right atrium, cardiac symptoms may occur because of venous return disturbance and tricuspid valve obstruction (6).

In general, there is a risk of blood flow disturbance due to the space-occupying effect of tumors in the cardiac lumen. Associated with this cardiac compression, other problems may arise, including pericardial effusion, low blood pressure, jugular vein distension, muffled heart sounds, arrhythmia, dyspnea, tachycardia, ascites, and increased respiratory effort $(7,10,22)$. In the aforementioned case of leiomyoma in a dog, the tumor caused atrioventricular block and induced brady- cardia. In the present case, symptoms such as muffled heart sound, anorexia, lethargy, dyspnea, and panting were noted, and the right atrial mass, pleural effusion, and pulmonary collapse were radiographically confirmed as the cause of the symptoms.

In general, right heart failure can occur due to right-sided filling pressure in the heart $(3,16,20)$. In the present case, pleural effusion, tumor, and severe tricuspid regurgitation were confirmed by diagnostic imaging evaluations, and pleural effusion and tumor were diagnosed by necropsy. It was thought that the tumor caused the increased right-sided filling pressure, leading to pleural fluid accumulation. The reason for the modified transudate in the pleural fluid was presumed to be due to cardiac hypertrophy and tumor necrosis. In dogs, the therapy for cardiac tumors is primarily aimed at treating arrhythmias and clinical signs of heart failure. However, the hemodynamic consequences of the neoplastic cardiac mass are often refractory to medical management without an effective anti-tumor treatment (19). In such cases, medication and thoracocentesis are performed without anti-tumor treatment according to the request of the owner to just reduce the overload to the heart and relieve the clinical symptoms. In fact, during the treatment period, there was an improvement of the symptoms and breathing difficulties, but the patient suddenly died during a hospital visit approximately 2 weeks after the first treatment. Because the 
cardiac tumor was not removed at all, its mass effect on the patient's body persisted and hydrothorax recurred. This cardiac compression and mechanical obstruction of blood flow $(10,19)$ was thought to be the main cause of the patient's sudden death. In the previous case of leiomyoma in a dog in which the tumor caused atrioventricular block, the authors reported a mass infiltrating the interventricular septum and the tricuspid annulus (5). In contrast, a mass infiltrating the right atrium and tricuspid valve was observed in the present case. Based on the location of the mass, the probability that the sudden death was due to supraventricular arrhythmia cannot be excluded.

As evidence of tumor was detected only in the heart, the clinical signs of the patient were attributed to cardiac tumors. Considering that the frequency of hemangiosarcoma is relatively high in canine heart tumors, the mass was considered to be an hemangiosarcoma until histopathological and immunohistochemical evaluations were performed $(2,8)$. However, the histopathologic results revealed an obvious histoid pattern, especially an interlacing pattern and spindle cells with blunt-ended nuclei, and these features can be seen when the tumor cells are derived from smooth muscle $(5,6,15)$. The mass was therefore diagnosed as a tumor of smooth muscle origin.

Histopathologic evaluation of this case showed tumor invasion in the myocardial and epicardial adhesion, but metastasis was not observed, and mitotic figures were rare. In addition, moderate cellular pleomorphism was confirmed. Encapsulation and non-encapsulation were also confirmed, but the rapid growth of the tumor was not detected. The degree of tumor differentiation was partially confirmed, and congestion and necrosis were found near the surrounding tissue invaded by the tumor. Comprehensively considered, the tumor was found to be a leiomyoma through histological examination. Notably, the tumor had some malignant elements as described above. However, it may be difficult to ascertain the type of the tumor by histopathological evaluation alone. Immunohistochemistry evaluation is necessary to confirm the diagnosis. Immunohistochemistry evaluation was performed using cytokeratin, vimentin, desmin, and smooth muscle actin for a definitive diagnosis $(5,6,18)$. Cytokeratin is a keratin-containing intermediate filament found in the intracytoplasmic cytoskeleton of epithelial cells. Vimentin is a protein that is a member of the intermediate filament family found in connective tissue. Desmin and smooth muscle actin are intermediate filaments found in muscle tissue and are markers that can be used to determine smooth muscle origin $(5,6)$. In this case, the histoid pattern, especially the interlacing pattern and spindle cells with blunt-ended nuclei, were identified. Neoplastic cells tested negative for cytokeratin, and positive for vimentin, desmin, and smooth muscle actin. The results indicated that the tumor was derived from mesodermal and smooth muscle cells. Therefore, the cardiac tumor, in this case, was finally diagnosed as a leiomyoma with characteristics of both leiomyoma and leiomyosarcoma, according to the histopathological and immunohistochemical findings.

According to the previous reports, there have been cases diagnosed with cardiac leiomyoma in veterinary medicine $(5,15)$ and medical science $(21)$. Strictly speaking, these cardiac tumors are likely to be of myocardial origin (not smooth muscle origin). However, histopathological immunostaining has displayed the pattern similar to leiomyoma in these reports. Likewise, our immunostaining pattern is the same as previous reports $(5,21)$ in histopathological evaluation. In present, histopathological evaluation using $\mathrm{IHC}$ is known to be accepted as the technique for definitive diagnosis in oncology. Therefore, we could be diagnosed with the same result (leiomyoma) in this patient. But, to the author's thought, further research or newly developed diagnostic technique might be needed to set a new definition of primary cardiac tumors considering the accurate origin.

\section{Conclusions}

To our knowledge, this is the first report of an intracardiac leiomyoma in Korea. Although leiomyoma is very rarely reported, it should be ruled out in the differential diagnosis when primary canine cardiac tumors are suspected. Despite the low incidence of this type of tumor, there is a considerable risk of sudden death due to intracardiac leiomyoma, and hence a definite diagnosis should be made based on immunohistochemistry evaluation. This report provides helpful information related to differential diagnosis and should be of interest to clinicians.

\section{Acknowledgements}

This work was supported by the National Research Foundation of Korea (NRF) grant funded by the Korea government (MSIT) (No. 2019R1F1A1060984).

\section{Conflicts of Interest}

The authors have no conflicting interests. 


\section{References}

1. Ahuja AK, Kumar S, Kumar A, Muddarangiah, Sobti D. Surgical management of vaginal leiomyoma in bitch. Int J Sci Env Technol 2017; 6: 2392-2395.

2. Brown NO, Patnaik AK, MacEwen EG. Canine hemangiosarcoma: retrospective analysis of 104 cases. J Am Vet Med Assoc 1985; 186: 56-58.

3. Cole PA. Association of canine splenic hemangiosarcomas and hematomas with nodular lymphoid hyperplasia or siderotic nodules. J Vet Diagn Invest 2012; 24: 759-762.

4. Fang BR, Ng YT, Yeh CH. Intravenous leiomyomatosis with extension to the heart: echocardiographic features: a case report. Angiology 2007; 58: 376-379.

5. Gallay J, Bélanger MC, Hélie P, Côté E, Johnson TO, Peters ME. Cardiac leiomyoma associated with advanced atrioventricular block in a young dog. J Vet Cardiol 2011; 13: 71-77.

6. Gidlewski J, Petrie JP. Therapeutic pericardiocentesis in the dog and cat. Clin Tech Small Anim Pract 2005; 20: 151-155.

7. Hammer AS, Couto CG, Filppi J, Getzy D, Shank K. Efficacy and toxicity of VAC chemotherapy (vincristine, doxorubicin, and cyclophosphamide) in dogs with hemangiosarcoma. J Vet Intern Med 1991; 5: 160-166.

8. Hong KH, Kim KS, Lee HY, Choi J. Urethral leiomyoma complicated with lower urinary tract obstruction in a dog. J Vet Clin 2012; 29: 181-185.

9. Kang MH, Kim DY, Park HM. Ectopic thyroid carcinoma infiltrating the right atrium of the heart in a dog. Can Vet J 2012; 53: 177181.

10. Kisseberth WC. Neoplasia of the heart. In: Withrow SJ, Vali DM, editors. Withrow \& MacEwen's small animal clinical oncology. St. Louis: Saunders Elsevier. 2007: 809-814.

11. Krotje LJ, Ware WA, Niyo Y. Intracardiac rhabdomyosarcoma in a dog. J Am Vet Med Assoc 1990; 197: 368-371.

12. Lamerato-Kozicki AR, Helm KM, Jubala CM, Cutter GC, Modiano JF. Canine hemangiosarcoma originates from hematopoietic precursors with potential for endothelial differentiation. Exp Hematol 2006; 34: 870-878.

13. Liptak JM, Forrest LJ. Soft tissue sarcomas. In: Withrow SJ, Vali DM, editors. Withrow \& MacEwen's small animal clinical oncology. St. Louis: Saunders Elsevier. 2007: 425-454.

14. Machida N, Hoshi K, Kobayashi M, Katsuda S, Yamane Y. Cardiac myxoma of the tricuspid valve in a dog. J Comp Pathol 2003; 129: 320-324.

15. Melo IS, Belo F, Gouveia R, Anjos R. Primary cardiac leiomyoma of the ventricular septum: a rare form of pediatric intracardiac tumor. Pediatr Cardiol 2012; 33: 649-651.

16. Natanzon A, Kronzon I. Pericardial and pleural effusions in congestive heart failure-anatomical, pathophysiologic, and clinical considerations. Am J Med Sci 2009; 338: 211-216.

17. Oksanen A. Haemangiosarcoma in dogs. J Comp Pathol 1978; 88: 585-595.

18. Radi ZA, Metz A. Canine cardiac rhabdomyoma. Toxicol Pathol 2009; 37: 348-350.

19. Ware WA. Cardiac neoplasia. In: Bonagura JD, Kirk RW, editors. Kirk's current veterinary therapy XII: small animal practice. Philadelphia: WB Saunders Elsevier. 1995: 873-876.

20. Ware WA, Hopper DL. Cardiac tumors in dogs: 1982-1995. J Vet Intern Med 1999; 13: 95-103.

21. Yahaya JJ. Primary leiomyoma of the left ventricle leading to sudden and unexpected death in a 74-year-old male: a case report. Egypt J Forensic Sci 2020; 10: 32.

22. Yamamoto S, Hoshi K, Hirakawa A, Chimura S, Kobayashi M, Machida N. Epidemiological, clinical and pathological features of primary cardiac hemangiosarcoma in dogs: a review of 51 cases. J Vet Med Sci 2013; 75: 1433-1441. 